\title{
SBDC: SMART BUILDING DATA CENTER FOR IOT, EDGE, AND 5G
}

\author{
Hassan Rajaei \\ Bhargav Kanumuri \\ Nishitha Narreddi \\ Computer Science Department \\ Bowling Green State University \\ Bowling Green, OH, 43403 \\ \{rajaei, bkanumu, nishitn\}@bgsu.edu
}

\begin{abstract}
Internet of Things (IoT) targets smart devices and applications to better assist the supported domains with intelligent technologies to increase the utilization of resources and users. Smart buildings constitute one such domain, where millions of devices generate terabytes of data demanding ultrahigh-speed computation and communication power for monitoring and analytical purposes. Latency in computation and communication is thus paramount. To meet such demand, this paper proposes a new architecture called Smart Building Data-Center (SBDC), an edge cloudlet node, furnishing high computing power and storage generated for vast IoT data in the building. In addition, this datacenter integrates a Mobile Edge Computing (MEC) of the Fifth Generation (5G) for all 5G wireless devices used in the building. Each SBDC is further connected to nearby smart buildings, cloud datacenters, and other networks in a Smart City. This paper describes the design, architecture, and network connectivity of the proposed system.
\end{abstract}

Keywords: IoT (Internet of Things), Mobile Edge Computing (MEC), Fifth Generation (5G), Cloud

\section{INTRODUCTION}

Advances in Internet of Things (IoT) (Borgia 2014) have broadened the vision and applications of connectivity to all smart objects, both physically and virtually. While data collection from all objects stands as the base of this technology, data processing, analytics, intelligence, and feedback are the key features. It is believed that by 2025 around 75 billion smart devices or more are anticipated to communicate with each other through the Internet (Ray 2017). These smart devices interact with each other and the supported infrastructure using various technological advances to furnish multiple intelligent services to their users. Examples of IoT applications include Smart Homes, Smart Buildings, Smart Cars, and Smart Healthcare. This paper focuses on Smart Buildings with high-speed computations and communication infrastructure.

Smart Buildings can be classified into various categories, however, we define them in three: Smart Residential Buildings, Smart Commercial Buildings, and Smart Hybrid Residential \& Commercial Buildings. The amount of traffic generated by these buildings is anticipated to exceed terabytes data (Wray 2019, Pincince 2015). Consequently, we are facing an explosion of ultra-big-data, generated, transported, processed, analyzed, and monitored in almost real-time. In other words, we are facing extraordinary circumstances which need extraordinary measures. Our solution is an integrated cloud computing datacenter at the edge with Mobile Edge Computing (MEC) of 5G inside the smart building. 
This solution can dramatically reduce the latency and increase efficiency and availability of the connected devices.

$5 \mathrm{G}$, the $5^{\text {th }}$ Generation of wireless networks, has extraordinary potential for IoT and smart devices. It has ultra-bandwidth of $10 \mathrm{Gbps}$, which is about 100 times faster than $4^{\text {th }}$ generation. $5 \mathrm{G}$ has better connectivity and decreased latency (Nuruddeen 2019) to alleviate one of the main challenges of IoT (Cnet 2019), which is connectivity among devices in the network. In addition, it facilitates ultra-large data traffic from devices and applications to the local datacenter and back in real-time.
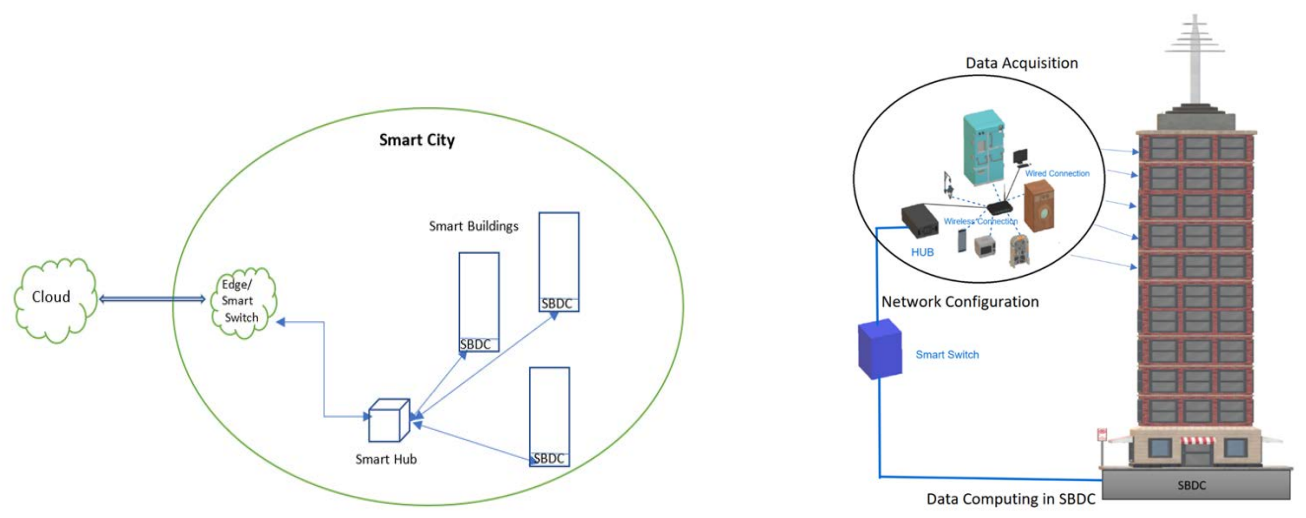

Figure 1: Overview of smart buildings' connectivity; a) A Smart city, b) Basic Data Accusation.

Figure 1 illustrates how smart buildings can be connected to each other and to a cloud in a smart city. Further, the figure shows how typical collecting nodes cluster with each other inside the building and the abstracting details focusing on the need for data collection and data processing to SBDC.

Different types of smart buildings can generate varying amounts of data. The amount of data generated can be overwhelming when scaling the buildings from small, medium, large, to ultra-large. MEC reduces network congestion by processing the data at the edge of the network (Wang et al 2017). The Smart Building Data-Center (SBDC) is configured to handle massive data with improved network connectivity and low latency as well as low network congestion using 5G and MEC, which will dramatically increase performance of the smart building. Traditionally, the data generated by a smart building is sent to a distant cloud or cloudlet for the necessary computing and storage purposes. Our solution lies inside the building, which can significantly reduce latency and increase efficiency of the building.

Smart objects with considerable intelligence are the cornerstone of IoT. The intelligence enables these objects to collect the needed information in real-time, process them quickly, and give feedback to the connected elements. IoT has three important phases: collection phase, transmission phase, and processing $\&$ utilization phase. Two additional phases are often added on the top of these three and are named as Analytics and AI, both of which add extra intelligent to the lifecycle of the data. A vital part of 5G, which is crucial for IoT as well, regards Mobile Edge Computing (MEC). 5G embraces Edge Computing and is Cloud Native, meaning the C-RAN (Cloud- Radio Access Network) fully supports cloud services at the edge, while sending heavy-duty services to the core cloud. As a result, our solution is fully integrated with 5G, MEC, Edge Computing, and Cloud. 
The rest of this paper is as follows: Section 2 presents related works, Section 3 describes SBDC echosystem, Section 4 describes Smart Buildings and their needs whereas Section 5 details network connectivity, and Sections 6 gives concluding marks and future works.

\section{RELATED WORK}

Minoli et al. (2017) described the requirements of smart buildings as an architecture with multiple layers. They defined "things" as sensors, alarm systems, and other IoT devices. Their layers include data acquisition, fog networking, data aggregation, data centralization, data analytics, and application layers. They consider residential and commercial buildings. The paper gives information about wireless technologies such as: long-range low bandwidth, long-range high bandwidth, short-range high bandwidth, and short-range low bandwidth. Types of wireless device include Z-wave, ZigBee, Wi-Fi, and Bluetooth (Kuzlu et al 2015).

Shua et al. (2018) describe hybrid optical network for a datacenter. The authors discuss about optical circuit switching (OCS), optical packet switching (OPS), and hybrid Top of the Rack (ToR) switches. They suggest that hybrid optical networks perform well when there are different types of data patterns in the network. The paper also gives simulation results about the behavior of their network. Dziyauddin et al. (2017) provides information about the communication between the sensors and IoT devices during emergencies. In this paper, the authors explained different types of sensors, wireless technologies, and network configuration used in a smart building. The authors proposed a framework for the smart emergency system in a smart building, and considered a fire as the emergency situation. They explained how communications can take place in emergency situations. Lin et al. (2014) introduced an Active Disaster Response System (ADRS) during emergencies. The ADRS informs the tenants during emergencies and performs tasks automatically.

Chen et al. (2012) provide a system for addressing configuration in a datacenter. The authors also discussed the connections between the servers and switches in the datacenter. They used a malfunction detection scheme to detect any link failures in the datacenter. The simulation results suggest the system can detect 3.8 million devices in 46 seconds. They implemented a datacenter auto-configuration using 64server BCube to respond in 30 seconds. Kalyanasundaram and Simmhan (2017) provided information about the ARM processors for Big-Data systems. They discussed ARM64 CPUs and the support for bigdata workloads. Authors performed a simulation study regarding the Intel's HI Bench suite of web and query by using apache Hadoop with a data size of $20 \mathrm{~GB}, 5$ web pages and 500 million tuples. The paper gave the basic idea about computational power of servers for large volumes of data. Emesowum et al. (2017) described network topologies in a datacenter and illustrated how to overcome network failures. The authors used different fat-trees such as hybrid fat-tree and reverse hybrid fat-tree, to overcome the challenges of fault-tolerant datacenter.

\section{A DATACENTER FOR SMART BUILDINGS}

Smart buildings generate massive amounts of data (Wray 2019, Pincince 2015, Lazarova-Molnar, and Mohamed 2017) which are often sent to a cloud or mobile edge for processing and storage purposes. However, there are great challenges to transfer such massive data back and forth to the cloud, or the edge, away from these buildings. Network delays and other unexpected jitters will significantly jeopardize the availability and performance of IoT devices scattered all over a smart building. Our solution is a Smart Building Data-Center (SBDC) that is inside the building to support most computational and storage needs of the intelligent services of the building. SBDC works as a Cloud Edge node which also connects to the core cloud when it is needed. In addition, SBDC also integrates a Mobile Edge Computing (MEC) for 5G devices used in the building. This solution is believed to dramatically reduce the network latency of the 
cloud and thus readily increase availability of the applications and services in the building. Figure 2 gives the growth rate of devices connected (in millions) in smart buildings whereas Table 1 demonstrates the data generated by several sizes of a commercial buildings (Statista 2019, Memoori 2018).

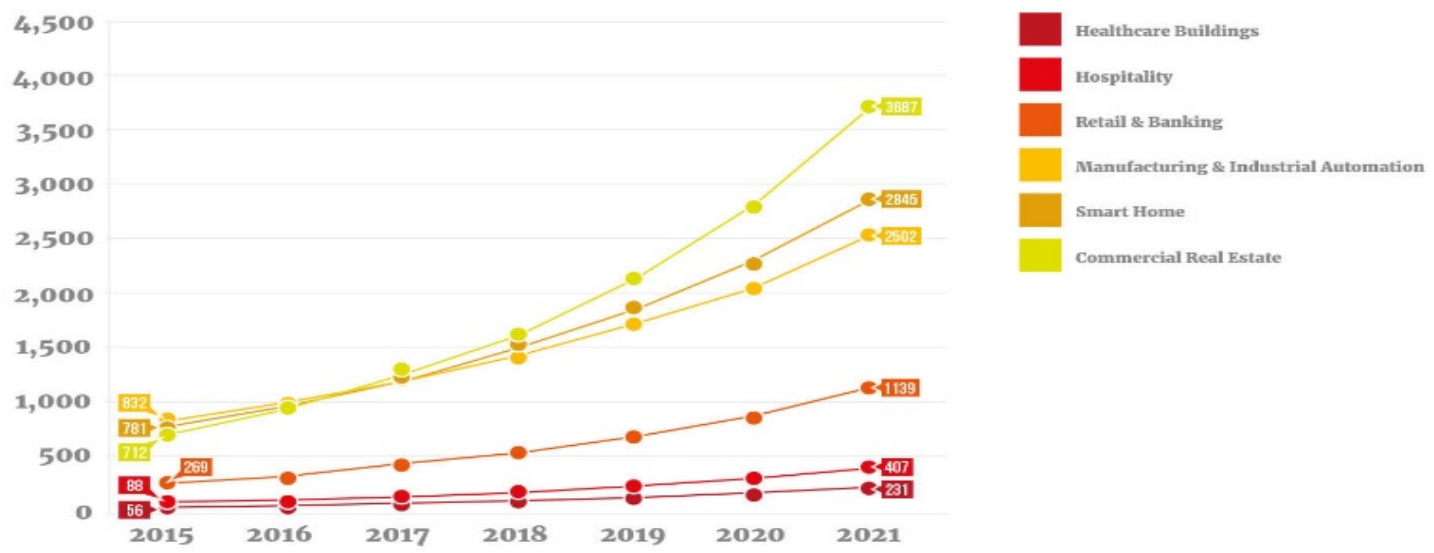

Figure 2: Growth rates of Connected Devices (in millions) in Smart Buildings (Memoori 2016).

Table 1: Data generated by smart commercial building according to:

(Memoori 2016, Hel-Net-Security 2019, and Memoori 2019).

\begin{tabular}{|l|c|l|}
\hline No. of Floors & Data Generated / Sec & Data Generated / Day \\
\hline 10 & 10.25 Terabytes & 885.6 petabytes \\
\hline 50 & 512.5 Terabytes & 44280 petabytes \\
\hline 100 & 1.025 petabyte & 88560 petabytes \\
\hline
\end{tabular}

\subsection{SBDC Architecture}

Figure 3 illustrates the architecture overview of the SBDC edge node. This datacenter has three general layers: 1) Application Layer; 2) The Edge Computing Layer; 3) The Infrastructure Layer.

\subsection{Application Layer}

Various IoT sensors collect data for the comfort of the users in a typical smart building. Moreover, multiple applications can have behavioral tracking, contextual awareness, analysis, and maintenance monitoring. The second phase of IoT (Borgia 2014, Rajaei et al 2018) ) transports the collected data to the processing units. The application layer is connected to the Application Support Interface in order to facilitate massive data-transfer between the sensors, applications, and the Edge Layer. 

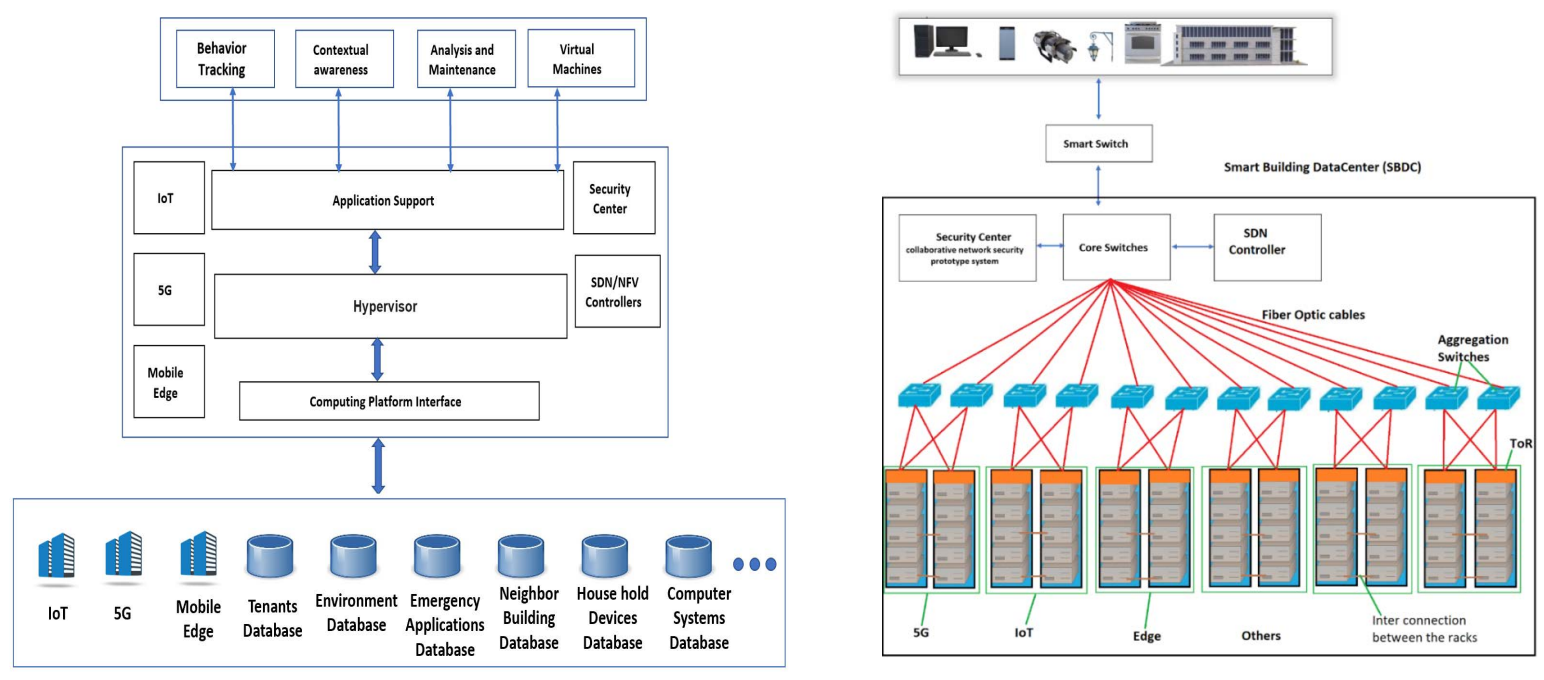

Figure 3: SBDC Architecture: a) the 3 layers, b) the Infrastructure layer.

\subsection{The Edge Computing Layer}

This layer contains the Application Support, IoT block, 5G block, Mobile Edge block, Security Center, SDN/NFV controllers, Hypervisor, and the interface to the hardware platforms. Further, it provides the abstraction view and virtualization services of the cloudlet to supply all applications in the building. The 5G and MEC are becoming the cornerstone of IoT, and are expected to increase in a number of connected devices. The Software Defined Networking (SDN) and Network Function Virtualization (NFV) blocks are needed for both the Edge Computing and the MEC for 5G networks. All abstraction software and virtualization of the edge cloud are installed and realized in this layer. Consequently, this layer is the brain of the SBDC datacenter.

\subsection{The Infrastructure Layer}

This layer is the heart of all hardware and the networks of SDBC. It includes rack computers, databases, storage, services, switches, networking services, among others. All data generated in a smart building eventually comes to this layer for processing, analysis, and storage. The results are sent back to the Edge Computing Layer which ultimately sent to the applications. This layer also stores information related to the tenants of this smart building, or the nearby buildings connected to a single SBDC.

The internal network of the datacenter has multiple configurations. It has a core switch block to connect the network infrastructure of the building as well as the main fat-tree networking of the hardware platform. Internal fat-tree network aggregation switches are basically configured as a Top-of-the-Rack (ToR) topology as shown in Figure 3b. In addition, for fault tolerance purpose, we use a Reversed Hybrid Fat-Tree (Emesowum et al 2017) to minimize any network and computational failure.

\subsection{Computing Power for Smart Buildings}

To realize the large value of data generated and processed by various smart buildings, we investigated and compiled Table 2 based to multiple sources (SiliconAngle 2019, Wharton 2018, Barroso et al 2013, Miller 2010). Table 2 shows how much processing power is required for each type of smart building. A small scale has 10 floors, medium scale contains 50 floors, large scale has 100 floors, and an ultra large scale contains more than 100 floors. For this table, we estimated the total amount of data generated and con- 
sumed by smart buildings during peak hours. As a result, the SBDC datacenter needs to be able to handle, process, and store greater than the peak hours capacity indicated by Table 2 .

Table 2: Processing power requirement of the different types of smart building.

\begin{tabular}{|l|l|l|}
\hline Building Types & Building Size & $\begin{array}{l}\text { Processing Power Needs } \\
\text { in peak hours /Sec }\end{array}$ \\
\hline Residential & Small & 5 Terabytes \\
\hline Residential & Medium & 25 Terabytes \\
\hline Residential & Large & 50 Terabytes \\
\hline Commercial & small & 10.25 Terabytes \\
\hline Commercial & Medium & 512.5 Terabytes \\
\hline Commercial & Large & 1.025 petabyte \\
\hline Hybrid & Small & 10.125 Terabytes \\
\hline Hybrid & Medium & 268.75 Terabytes \\
\hline Hybrid & Large & 537.5 Terabytes \\
\hline
\end{tabular}

\section{SMART BUILDINGS}

Smart buildings are regarded as an essential application of IoT, where other examples include smart healthcare, smart cars, and smart cities. Data generated by the smart buildings are often sent to a cloud or a nearby datacenter for processing. In contrast, we designed a dedicated cloudlet called Smart Building Data-Center (SBDC) located at the basement of the building, at the edge of the applications. Advantages include low latency, improved performance, and high processing power. Different smart buildings generate different data traffic, and thus require different processing power and network infrastructure.
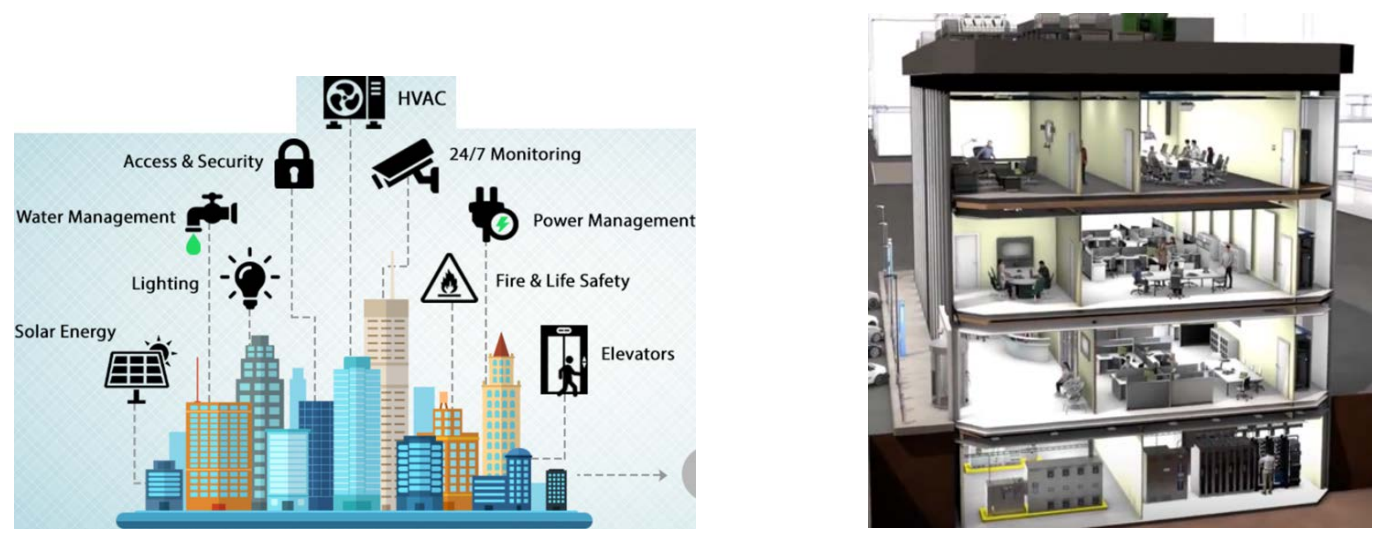

Figure 4: IoT \& Smart Building a) Overview, b) use of datacenter. 


\subsection{Types of Smart Building}

We consider three types of smart buildings: residential, commercial, and hybrid buildings. Key features of these buildings include: maximizing efficiency and the productivity, safe and secure environment, highspeed network connectivity, IoT enabled monitoring environment, and assisting users' day-to-day life. To do so, the building should accommodate high-performance computing and communication infrastructure connected to all floors and to the datacenter in the building. Five essential supervisions of smart buildings include: environmental monitoring, visual management, communication management, security integration, and network connectivity.

\subsubsection{Residential Smart Buildings}

These buildings provide automated services to their residents. We assume there are five smart homes in each floor, where the number of floors can range from ten to hundreds. Each building can either have a SBDC datacenter inside or use one connected to the next building as was shown in Figure 1. Each SBDC provides processing power and data storage for the residents. Figure 5 gives an overview of Smart Residential Building, its datacenter, and the network connectivity. Each floor (Figure 5b) has five units connected to a high-speed hub and a smart switch and connects to the SBDC. The network backbone of a smart building use multi-gigabits fiber-optic cables to connect each floor and home to the datacenter.
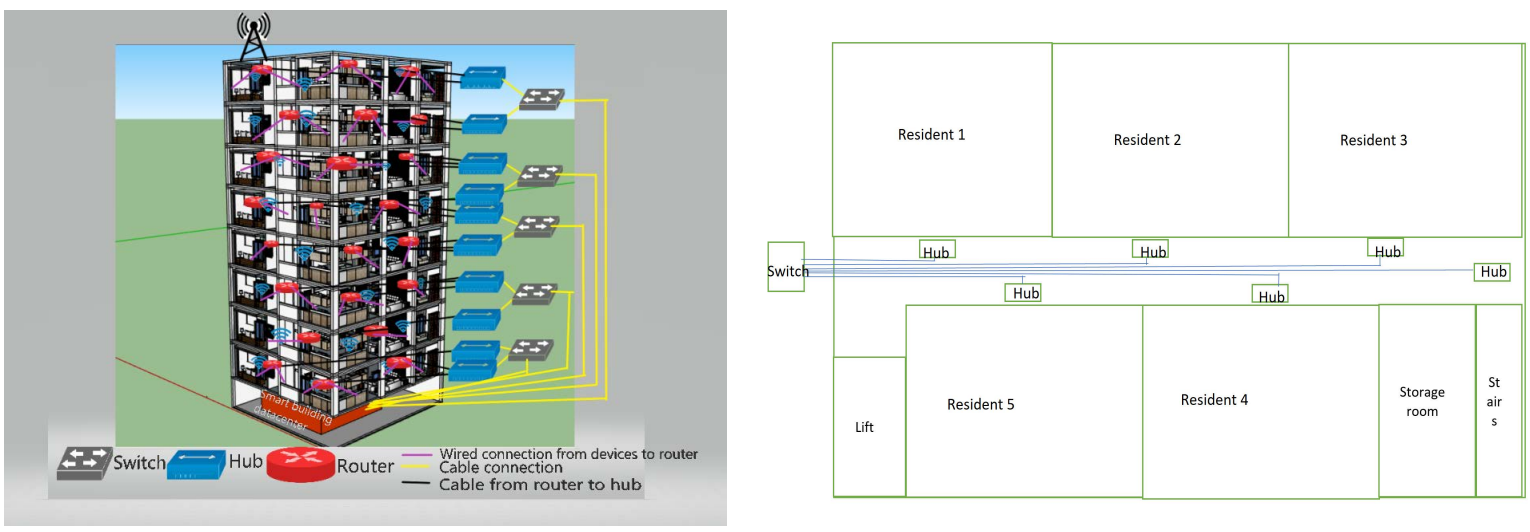

Figure 5: Smart Residential Building a) Connectivity structure of 10 floors, b) Each floor structure plan.

\subsubsection{Commercial Smart Buildings}

The size of the buildings varies from ten floors to over one hundred floors, each equipped with high-speed communication and computation devices. Each floor could accommodate up to 200 hundred employees, assuming two blocks are on each floor. The data generated by the smart commercial buildings are often 20 times larger than residential ones. Further, we assume every employee has 3 systems, each needs high processing power to run multiple applications at the same time. The networking infrastructure is enhanced, compared to residential ones, to accommodate a large volume of traffic. Numerous wireless communications technologies are used such as Wi-Fi, Bluetooth, ZigBee, Z-Wave, and 5G. The network connectivity is enhanced with additional hubs and switches to handle large data traffic. The SBDC datacenter furnishes an ultrahigh-speed computing cloudlet at the edge of its applications. Figure 6 illustrates a smart commercial building with SBDC support. Kanumuri et al (2019) gives detailed information and floorplan about smart buildings. Numerous configurations of floorplan exist for any commercial build. Our work focuses on the computational and communication power for each floorplan with typical number of employee in that unit to quantify our performance evaluation models. 

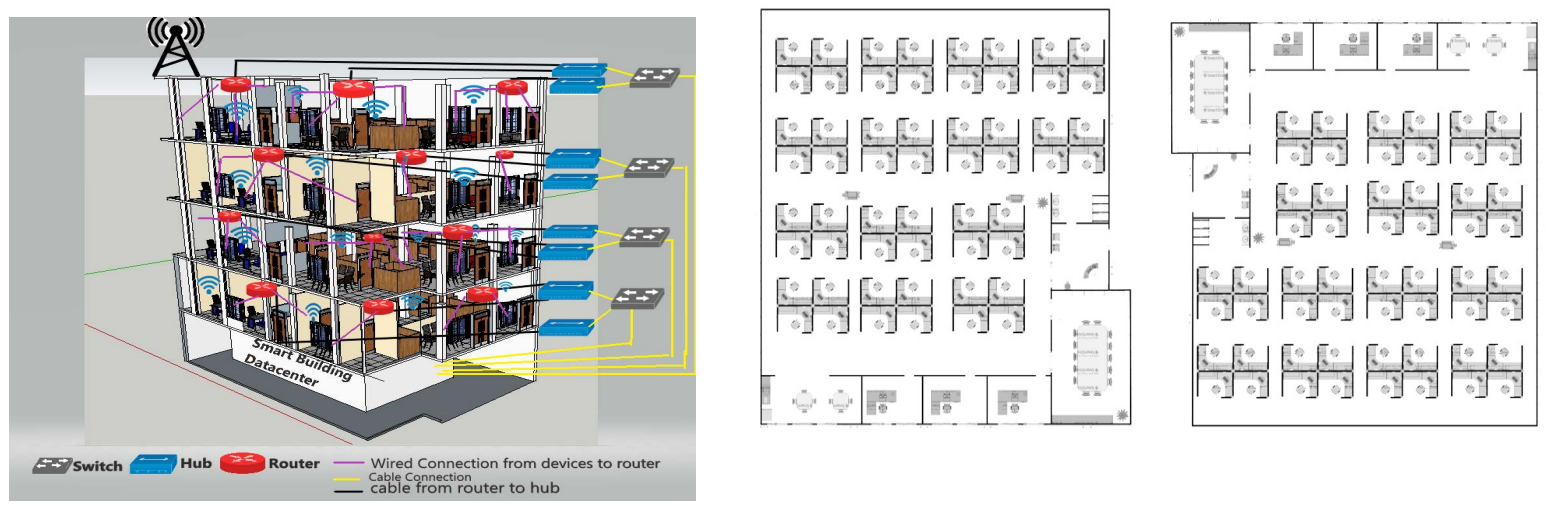

Figure 6: Smart Commercial Building a) Connectivity and layout, b) Basic structure of a floor plan.

\subsubsection{Hybrid Commercial and Residential Smart Buildings}

Hybrid buildings are a mix of the residential and commercial buildings. Utilization of communication and computation needs in SBDC is different from the previous two types. The commercial units need the highest power and data traffic during the daytime, whereas the residential units contrast, and may have these needs at night. As a result, the SBDC power will be used continuously in these buildings. In particular, during the daytime, the workload of the commercial units will be very high, while the workload of the residential ones will be low. At night, the situation reverses and the workload of the residential building will become higher. This characteristic will give an efficient utilization scheme to optimize SBDC resources.

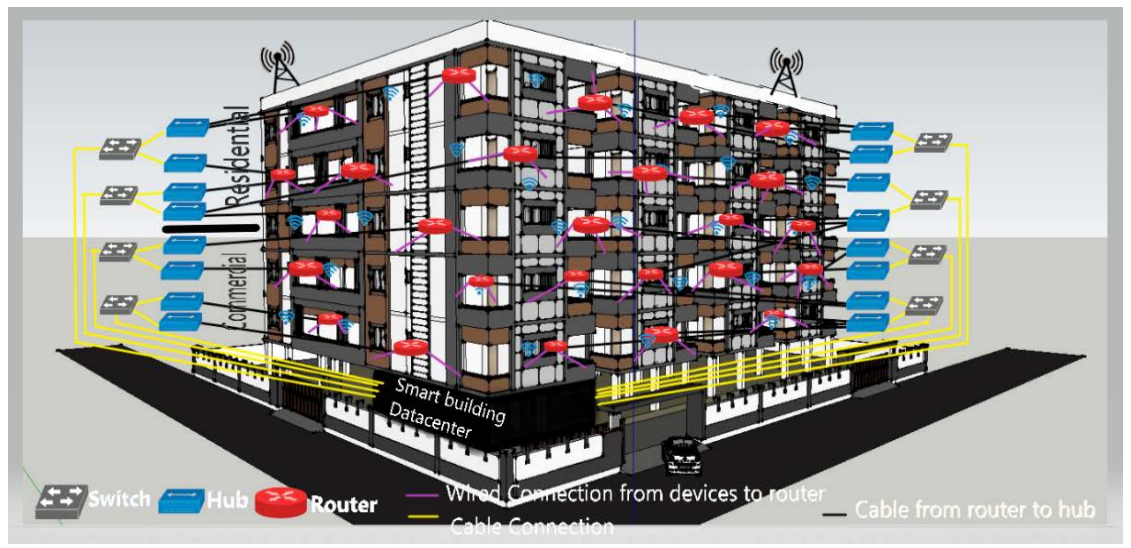

Figure 7: A Hybrid Commercial and Residential Building.

\section{NETWORK CONNECTIVITY}

Network connectivity plays an essential role in smart buildings. Performance and reliability of these buildings are heavily dependent on robust network configurations and ultrafast data-traffic transportation. IoT implements additional smart devices which require speedy and reliable traffic. As a result, smart buildings must accommodate ultrahigh bandwidth using both wired and wireless networks. We integrate 5G in our design based on two 5G key features: a) ultrahigh bandwidth, b) use of MEC (Mobile Edge Cloud). SBDC supports a MEC for $5 \mathrm{G}$ devices in the building. 


\subsection{G Network Configurations and Integration}

\subsubsection{Residential Networks}

Figure 8 illustrates the network configuration in one residential home mixed with $5 \mathrm{G}$ and smart switches. We consider a living room, kitchen, 3 bedrooms, and some other facilities. In addition to $5 \mathrm{G}$, there are other IoT devices and sensors connected with various wireless technologies such as Wi-Fi, ZigBee, ZWave, and Bluetooth. The SBDC edge node is in the basement for security reasons, and is connected to high-speed switches, hubs, routers, and sensors. The dataflow for processing to the datacenter follows: sensors $\rightarrow$ routers $\rightarrow$ hubs $\rightarrow$ switches $\rightarrow$ SBDC, and in reverse the dataflow goes SBDC $\rightarrow$ switches $\rightarrow$ hubs $\rightarrow$ routers $\rightarrow$ sensors or IoT devices.
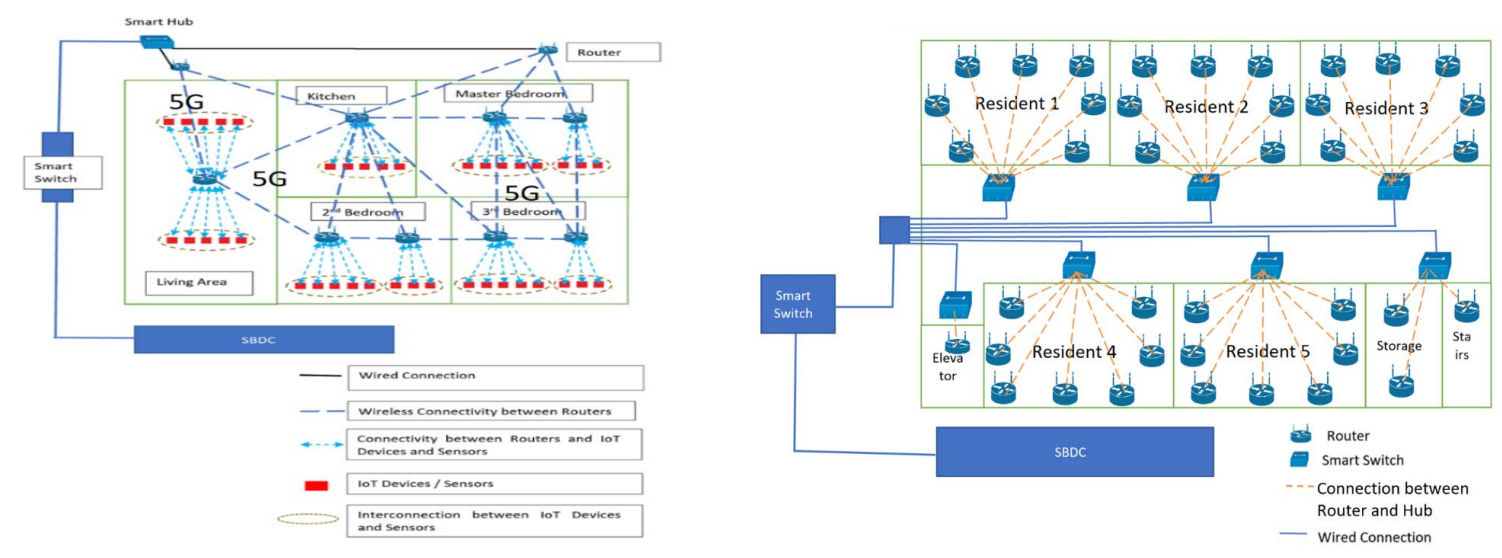

Figure 8: 5G network integration; a) one smart home, b) one floor of a residential building.

\subsubsection{Commercial Networks}

We assume 200 employees are on each floor of a commercial building. Further, each employee has 3 systems. There are different offices in each block including a conference room and service area. The workload of these buildings is higher compared with residential ones. Figure 9 shows the network configuration for one block, each containing a manager's room, employee rooms, conference rooms, and a cafeteria. Each floor has one main switch that is connected to SBDC via fiber-optic cables.
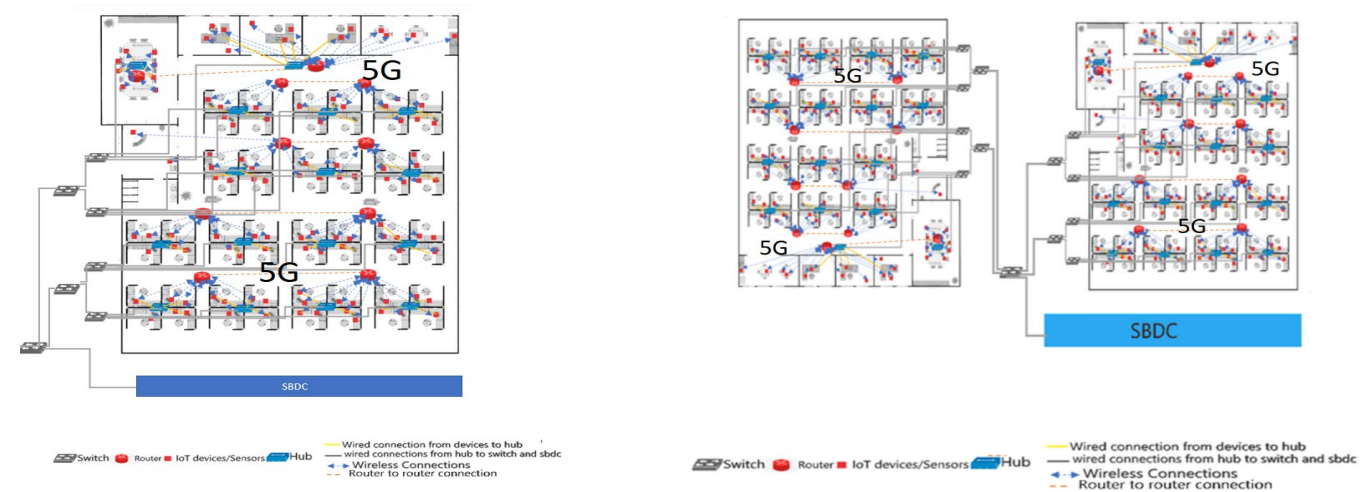

Figure 9: 5G network integration; a) one smart commercial block, b) one floor commercial building. 


\section{CONCLUDING REMARKS AND FUTURE WORK}

Smart buildings are the heart of smart cities of future societies. They generate tremendous amount of data each day and require immense computing and communication power almost instantly. This is an extraordinary challenge which requires extraordinary methods. One such method is designed and described in this paper. Our solution includes an edge computing node called SBDC which is inside a smart building. Further, it is integrated with $5 \mathrm{G}$ and MEC, as well as supporting IoT devices. The paper described the details of the networking infrastructure of smart buildings and the architecture of the edge cloudlet. Through the compiled research data, we argued the necessity of having an edge cloud center in the building and the necessary network infrastructure of the building. What is missing in this paper is the evaluation, robustness, and performance analysis of the proposed architecture and its ecosystem. In our future study, multiple issues are planned to shed light on not only our system, but also the data presented in this paper as well. Our plan includes detailed simulation studies and an analysis of SBDC, with multiple configurations, scalability issues, and the fault tolerance of the smart buildings. In addition, certain cases of failure such as communication shortages, disaster recovery, fire outbreak, etc., need to be included in such a study.

\section{REFERENCES}

Barroso, L. A., Clidaras, J., Hoelzle, U., \& Holzle, U. (2013). Datacenter as a Computer An Introduction to the Design of Warehouse-Scale Machines. San Rafael: Morgan \& Claypool Publishers.

Basu, D, Moretti, G, Gupta, S, \& Marsland S, (2013), "Wireless Sensor Network based Smart Home: Sensor selection, deployment and monitoring", In Proc. of IEEE Conf, Sensors Applications Symposium (SAS 2013), pp. 49-54

Borgia, Eleonora, (2014), “The Internet of Things: Key features, applications, and open issues", Elsevier Journal of Computer Communication, 54 (2014) 1-13.

Chen, K., Guo, C., Wu, H., Yuan, J., Feng, Z., Chen, Y., ... Wu, W. (2012). DAC: Generic and Automatic Address Configuration for Data Center Networks. IEEE/ACM Transactions on Networking, 20(1), 84-99.

Cnet, 2019, "Wireless Broadband-Like 5G To Drive IoT Innovations Over Next Decade," MITechNews, 04-Jan-2018.[Online].Available:https://mitechnews.com/internet-of-things/wireless-broadband-like5g-drive-iot-innovations-next-decade/. [Accessed: 30-Sep-2019].

Dziyauddin, R. A., Shah, A. I. J., Omar, M. H., Zamri, N. H., Latiff, L., Noor, N. M., \& Ahmad, N. S. (2018). Smart Fire Emergency System for Buildings: A Review. 2018 2nd International Conference on Telematics and Future Generation Networks (TAFGEN).

Emesowum, H., Paraskelidis, A., \& Adda, M. (2017). Fault tolerance capability of cloud data center. 2017 13th IEEE International Conference on Intelligent Computer Communication and Processing (ICCP).

Help Net Security, June 2019, “41.6 billion IoT devices will be generating 79.4 zettabytes of data in 2025”, Retrieved from https:/www.helpnetsecurity.com/2019/06/21/connected-iot-devices-forecast/.

Kalyanasundaram, J., \& Simmhan, Y. (2017). ARM Wrestling with Big Data: A Study of Commodity ARM64 Server for Big Data Workloads. 2017 IEEE 24th International Conference on High Performance Computing (HiPC).

Kanumuri, B, Narreddi, N, Rajaei, H. (2019). Smart Building Data Center, for Edge, IoT, and 5G. Technical Report, Dept. Computer Science, BGSU, Ohio. 
Kuzlu, M., Pipattanasomporn, M., \& Rahman, S. (2015). Review of communication technologies for smart homes/building applications. 2015 IEEE Innovative Smart Grid Technologies - Asia (ISGT ASIA).

Lazarova-Molnar, S., and Mohamed, N. (2017). Collaborative data analytics for smart buildings: opportunities and models. Cluster Computing, 22(S1), 1065-1077.

Liang L, Huang L, Jiang X, \& Yao Y, (2008), "Design and Implementation of Wireless Smart-Home Sensor Network based on ZigBee protocol”, in Proc. of ICCCAS'08 IEEE conf. on -Smart Home \& ZigBee

Lin, C.-Y., Chu, E., Ku, L.-W., \& Liu, J. (2014). Active Disaster Response System for a Smart Building. Sensors, 14(9), 17451-17470.

Miller, R. (2010, February 17). How Intel Manages 100,000 Servers. Retrieved from https:/www.datacenterknowledge.com/archives/2010/02/17/how-intel-manages-100000-servers.

Memoori 2016, "The Internet of Things in Smart Commercial Buildings 2016 to 2021". (n.d.). Retrieved from https://memoori.com/portfolio/internet-things-smart-commercial-buildings-2016-2021/

Memoori 2018, "The Internet of Things in Smart Commercial Buildings 2018 to 2022" (n.d.). Retrieved from https://memoori.com/portfolio/internet-things-smart-commercial-buildings-2018-2022/

Minoli, D., Sohraby, K., \& Occhiogrosso, B. (2017). IoT Considerations, Requirements, and Architectures for Smart Buildings - Energy Optimization and Next Generation Building Management Systems. IEEE Internet of Things Journal, 1-1.

Nuruddeen, A. U. (2019). An Overview: Internet of Things, 5G Communication System and Cloud Computing.

Pincince, T. (2015). Smart building: Building bridges between IT and facilities. Retrieved from https://www.oreilly.com/ideas/smart-building-building-bridges-between-it-and-facilities.

Rajaei, H. Mirzaei, F. (2018). IoT, Smart Homes, and ZigBee Simulation, SpringSim-CNS 2018, April 15-18, Baltimore, MD, USA

Ray, S. (2017). System-on-chip security assurance for IoT devices: Cooperations and conflicts. 2017 IEEE Custom Integrated Circuits Conference (CICC).

Shu, Y., Yan, S., Jackson, C., Kondepu, K., Salas, E. H., Yan, Y., ... Simeonidou, D. (2018). Programmable OPS/OCS hybrid data centre network. Optical Fiber Technology, 44, 102-114.

SiliconAngle 2019, "Smart home data is flowing for the Samsung SmartThings platform", Sept, Retrieved from https://siliconangle.com/2019/09/17/smart-home-data-is-flowing-for-the-samsungsmartthings-platform-sumoilluminate/.

Statista 2019, "Data collected by smart buildings worldwide 2010-2020". (n.d.). Retrieved from https://www.statista.com/statistics/631151/worldwide-data-collected-by-smart-buildings/.

Wang, S., Zhang, X., Zhang, Y., Wang, L., Yang, J., \& Wang, W. (2017). A Survey on Mobile Edge Networks: Convergence of Computing, Caching and Communications. IEEE Access, 5, 6757-6779.

Wray, Sarah, 2019 "Intel bids for 'smartest building in the world' title with new office". (n.d.). Retrieved from https://www.smartcitiesworld.net/news/news/intel-bids-for-smartest-building-in-the-world-titlewith-new-office--4747.

Wharton, U of Pen, Operations Management, Mar 2018, "How to Turn 'Data Exhaust' into a Competitive Edge". Retrieved from https://knowledge.wharton.upenn.edu/article/turn-iot-data-exhaust-nextcompetitive-advantage/. 


\section{AUTHOR BIOGRAPHIES}

HASSAN RAJAEI is a Professor of Computer Science at Bowling Green State University, Ohio. His research interests include Internet of Things (IoT), Cloud Computing, High Performance Computing (HPC), computer simulations and distributed simulations with application of Wireless Communications \& Networks, and Virtual Environments. Dr. Rajaei received his Ph.D. from Royal Institute of Technology, KTH, Stockholm, Sweden and he holds a MSEE from Univ. of Utah, and a BS from University of Tehran. His email address is rajaei@bgsu.edu.

BHARGAV KANUMURI has been a graduate student of Computer Science at BGSU. He took multiple courses in distributed systems with Dr. Rajaei. For his MS Project, he conducted research on IoT-based Smart Buildings using MEC and 5G. He received an MS Degree in Computer Science in Fall 2019. His email address is bkanumu@bgsu.edu.

NISHITHA NAREDDI is a graduate student of Computer Science at BGSU. She took multiple courses in distributed systems with Dr. Rajaei. For her MS Project, she conducted research on IoT-based Smart Buildings using MEC and 5G. She is expecting to receive an MS Degree in Computer Science in Spring 2020. Her email address is nishitn@bgsu.edu. 